\title{
The energy dependence of antiparticle to particle ratios in high energy $p p$ collisions
}

\author{
Wang Jiang-ling ${ }^{1}$ Chen Gang ${ }^{1,2 *}$ Li Hai-jun ${ }^{1}$ and Li Di-kai ${ }^{1}$ \\ ${ }^{1}$ School of Mathematics and Physics, \\ China University of Geosciences, Wuhan 430074, China. \\ ${ }^{2}$ Key Laboratory of Quark and Lepton Physics (MOE), \\ Central China Normal University, Wuhan 430079, China \\ chengang1@cug.edu.cn
}

\begin{abstract}
The energy dependence of antiparticle to particle ratios in $p p$ collisions of high energy is studied using the PACIEA and DCPC model. The yield ratios of antimatter and matter for different masses are measured at various energies. It is found that the yield ratios of antimatter and matter increase with the increase of the c.m energy of $p p$ collisions until they gradually approach to 1 after the c.m energy is more than $200 \mathrm{GeV}$. The distribution of transverse momentum also has significant dependence on the energy and mass, i.e the average transverse momentum increase when the c.m energy of $p p$ collisions increase. The model results are compatible with the STAR preliminary datum.

PACS numbers: 25.75.-q, 24.85.+p,24.10.Lx
\end{abstract}

\section{INSTRUCTIONS}

The investigation of antimatter has great importance in nuclear and particle physics, astrophysics, and cosmology [1]. Matter and antimatter existed in equal abundance during the initial stage of the universe. However, after a series of evolution, now the amount of antimatter is far less than the amount of matter. Since the evolution of the initial fireball created in relativistic heavy-ion collisions is similar to the initial stage of the universe, the relativistic heavy-ion collision experiment is the appropriate means of antimatter investigation.

In 1928, based on the application of symmetry rules of quantum mechanics, the British physicist Dirac predicted that each particle had a corresponding antiparticle, which has opened the prologue of antimatter research [2]. Then, in 1932, American scientists Anderson found positrons in the cosmic rays, which caused a stir in the scientific community [3], in the United States in 1955, Segre and Chamberlain found antiprotons in Berkeley proton synchrotron experiments [4], then in 1956, Cork B. et al. found the anti-neutron when accelerating the protons to $6.2 \mathrm{GeV}$ in Tevatron accelerator [5]. The discovery of antimatter on the scale of nucleus, was started with the measurement of anti-hydrogen in 1995, and finally in January 17, 2010, the scientists captured 38 anti-hypertritium in LHC, CERN [6].

Recently, the STAR collaboration has reported their results in measuring the Au-Au collisions at top RHIC energy region. They found $70 \pm 17$ anti-tritium and $157 \pm 30$ tritium in $\mathrm{Au}-\mathrm{Au}$ collisions at $\sqrt{s}=200 \mathrm{GeV}$, with $89 \times 10^{6}$ events and $22 \times 10^{6}$ central collision events. ALICE collaboration also published the yield of anti-deuterium is approximately $6 \times 10^{-5}$, in $7 \mathrm{TeV} p p$ collisions $[7,[8]$. On the other hand, the theoretically research is generally divided into two steps. First, select a model to calculate the nucleons and hyperons, such as the transport model. Then, use the phase-space coalescence model [9] or statistical model [10] to calculate the light (anti-)nuclei. Some scientists use the coalescence+blast-wave method [11] and UrQMD hydro hybrid model+thermal model [12] to do the theoretical research on the production of light nuclei and hyper nuclei in $\mathrm{Au}-\mathrm{Au}$ and $\mathrm{Pb}-\mathrm{Pb}$ collisions at relativistic energy.

Based on PACIAE [13], we proposed a new model, dynamically constrained phase-space coalescence model (DCPC), to study light (anti-)nuclear. Using this model, we have investigated the production of light(anti)nuclei in relativistic $p p$ collisions [1], the production of light (anti)nuclei and (anti)hypertritium in $\mathrm{Au}-\mathrm{Au}$ collisions [14], and their centrality dependence [15] and scaling feature [16]. The consistency of the results of the model and the corresponding experimental data shows that PACIAE+DCPC model is an effective method which can be used to describe the production of light (anti)nuclei and (anti)hypernucleus in relativistic heavy ion collisions [1, 15, 16].

In this paper, we firstly calculate the hadronic final state using the PYTHIA [17] and PACIAE model in the non-single diffractive (NSD) $p p$ collisions at different c.m energies. Then we can generate the light(anti)nuclei using the dynamically constrained phase-space model to investigate the energy dependence of antiparticles production in high energy $p p$ collisions. In section 2, we briefly describe our model. In section 3, our numerical results are presented and in section 4 , we give a short summary.

* Email:chengang1@cug.edu.cn 


\section{MODELS}

PYTHIA is a model for high energy hadron-hadron $(h h)$ collisions [17]. The parton and hadron cascade model, PACIAE [13], is based on PYTHIA. In the PYTHIA model a hh collision is decomposed into parton-parton collisions. The hard parton-parton collision is described by the lowest leading order perturbative QCD (Lo-pQCD) partonparton interactions with the modification of parton distribution function in a hadron. The soft parton-parton collision, which is non-perturbative phenomenon, is considered empirically. The initial- and final-state QCD radiations and the multiparton interactions are also taken into account. So the consequence of a $h h$ collision is a partonic multijet state composed of di-quarks (anti-diquarks), quarks (antiquarks), and gluons, besides a few hadronic remnants. It is then followed by the string construction and fragmentation and a hadronic final state for a $h h$ (pp) collision is eventually obtained.

The PACIAE model is mainly different from the PYTHIA as follows:

1. The string fragmentation is switched-off temporarily and the di-quarks (anti-diquarks) are broken randomly into quarks (antiquarks). So the consequence of pp collision is a initial state of quarks, antiquarks, and gluons, besides a few hadronic remnants. This partonic initial state is regarded as the quark-gluon matter (QGM) formed in the relativistic pp collisions.

2. The parton rescattering is introduced. In this stage the rescattering among partons in QGM is calculated by the $2 \rightarrow 2$ Lo-pQCD parton-parton interaction cross sections [18]. However, a $K$ factor is introduced to include the higher order and non-perturbative corrections. The effective strong coupling constant, $\alpha_{s}$, is assumed to be 0.47 . A parton colour screen mass $\mu=0.63 \mathrm{GeV}$ is introduced to avoid the divergence. By integrating the differential cross sections above the total cross section of the parton collision is obtained. Then the parton rescattering is simulated by Monte Carlo method.

3. The hadronization is then proceeded after parton rescattering. The partonic matter can be hadronized by the Lund string fragmentation regime [17] and/or the phenomenological coalescence model [13].

4. At last the hadron rescattering is added. In this stage the hadronic matter after hadronization proceeds rescattering. This rescattering is dealt with by the usual two-body collision method [19], until the hadronic freeze-out is reached(the exhaustion of parton-parton collision pairs).

From quantum statistical mechanics [20], one can not precisely define both position $\vec{q} \equiv(x, y, z)$ and momentum $\vec{p} \equiv\left(p_{x}, p_{y}, p_{z}\right)$ of a particle in six dimension phase space, because of the uncertainty principle, $\Delta \vec{q} \Delta \vec{p} \geq h^{3}$. One can only say this particle lies somewhere within a six dimension quantum "box" or "state" of volume of $\Delta \vec{q} \Delta \vec{p}$. A $h^{3}$ volume element in the six dimension phase space corresponds to a state of the particle [20]. Therefore, one can estimate the yield of a single particle by

$$
Y_{1}=\int_{H \leq E} \frac{d \vec{q} d \vec{p}}{h^{3}} .
$$

Similarly, the yield of $\mathrm{N}$ particles cluster can be estimated by

$$
Y_{N}=\int \ldots \int_{H \leq E} \frac{d \vec{q}_{1} d \vec{p}_{1} \ldots d \vec{q}_{N} d \vec{p}_{N}}{h^{3 N}} .
$$

In the dynamically constrained phase space coalescence model, the yield of $\overline{\frac{3}{\Lambda} H}$, for instance, is assumed to be

$$
\begin{aligned}
Y_{\overline{\frac{3}{\Lambda} H}}= & \int \ldots \int \delta_{123} \frac{d \vec{q}_{1} d \vec{p}_{1} d \vec{q}_{2} d \vec{p}_{2} d \vec{q}_{3} d \vec{p}_{3}}{h^{9}}, \\
\delta_{123}= & \left\{\begin{array}{c}
1 \text { if } 1 \equiv \bar{p}, 2 \equiv \bar{n}, 3 \equiv \bar{\Lambda}, \text { and combination; } \\
m_{0} \leqslant m_{i n v} \leqslant m_{0}+\Delta m ; \\
\left|\vec{q}_{12}\right| \leqslant D_{0}, \quad\left|\vec{q}_{13}\right| \leqslant D_{0}, \quad\left|\vec{q}_{23}\right| \leqslant D_{0} ; \\
0 \text { otherwise, }
\end{array}\right.
\end{aligned}
$$

where

$$
m_{i n v}=\left[\left(E_{1}+E_{2}+E_{3}\right)^{2}-\left(\vec{p}_{1}+\vec{p}_{2}+\vec{p}_{3}\right)^{2}\right]^{1 / 2}
$$

$m_{0}$ and $D_{0}$ stand for the rest mass and diameter of $\overline{\frac{3}{\Lambda} H}, \Delta m$ refers to the allowed uncertainty, and $\left|\vec{q}_{i j}\right|$ is the distance between particles $i$ and $j$. 
The integral over continuous distributions in Eq. (3) should be replaced by the sum over discrete distributions as the hadron position and momentum distributions from transport model simulation are discrete. In a single event of the final hadronic state from transport model simulation, the system of $\overline{3} H$ can be identified by the configurations of $\bar{p}, \bar{n}$, and $\bar{\Lambda}$. This configuration can be expressed as

$$
C_{\bar{p} \bar{n} \bar{\Lambda}}\left(\Delta q_{1}, \Delta q_{2}, \Delta q_{3} ; \vec{p}_{1}, \vec{p}_{2}, \vec{p}_{3}\right)
$$

where the subscripts $1,2,3$ stand for the $\bar{p}, \bar{n}$ and $\bar{\Lambda}$, and $\Delta q_{i}=\left|\vec{q}_{i}-\vec{q}_{c}\right| \quad(i=1,2,3)$ refers to the distance between $i$ th particle and the center-of-mass of $\bar{p}, \bar{n}$, and $\bar{\Lambda}$ for instance. Correspondingly, the third constraint (diameter constraint) in Eq. (4) is replaced by

$$
\Delta q_{i} \leqslant R_{0}, \quad(i=1,2,3)
$$

where $R_{0}$ refers to the radius of $\overline{\frac{3}{\Lambda} H}$.

Each configuration above contributes an partial yield of

$$
Y_{123}= \begin{cases}1 & \text { if } m_{0} \leqslant m_{i n v} \leqslant m_{0}+\Delta m \\ & q_{1} \leqslant R_{0}, \quad q_{2} \leqslant R_{0}, \quad q_{3} \leqslant R_{0} \\ 0 & \text { otherwise }\end{cases}
$$

to the $\overline{3} H$. Its total yield in a single event is then the sum of above partial yield over the configurations of Eq. (66) and its combination. An average over events is required at the end.

\section{THE RESULTS}

In the PYTHIA and PACIAE simulations, we assume that the hyperons heavier than $\Lambda$ decay already. The model parameters were fixed on the default values given in PYTHIA model, except the $K$ factor and the parameters of $\operatorname{parj}(1), \operatorname{parj}(2)$, and parj(3) were roughly fitted to the STAR data of $\Xi^{+}, \Xi^{-}, \Lambda$, and $\bar{\Lambda}$ in NSD $p p$ collisions at $\sqrt{s}=$ $200 \mathrm{GeV}$ [1]. Here the $K$ factor is introduced to consider the higher order and non-perturbative QCD corrections. $\operatorname{parj}(1)$ is the suppression of diquark-antidiquark pair production compared with quark-antiquark production. parj(2) is the suppression of $s$ quark pair production compared with $u(d)$ pair production. parj(3) is the extra suppression of $s$ diquark production compared with the normal suppression of $s$ quarks. The fitted parameters of $K=3$ (default value is 1 or 1.5$), \operatorname{parj}(1)=0.15(0.1), \operatorname{parj}(2)=0.38(0.3)$, and $\operatorname{parj}(3)=0.45(0.4)$. We use the PYTHIA and PACIAE transport model to generate the hadronic final states, and use the DPCP model to generate the light (anti)nuclei, respectively, then to study the characteristics of them.

In Figure 1, the yield ratios of antiparticles to corresponding particles in $p p$ collisions at $200 \mathrm{GeV}$ and $7 \mathrm{TeV}$ are given, including $\pi^{-} / \pi^{+}, K^{-} / K^{+}, \bar{p} / p, \bar{\Lambda} / \Lambda, \overline{\Xi^{-}} / \Xi^{-}, \overline{\Omega^{-}} / \Omega^{-}, \bar{d} / d, \overline{{ }^{3} H e} /{ }^{3} H e$, and $\frac{\overline{3} H}{\Lambda} / \frac{3}{\Lambda} H$, respectively. For comparison, the experimental results are also drawn in the Figure 1, as the dots representing STAR data [21] and the solid triangle is ALICE data [22]. From the figure 1, it can be seen that, all ratios of antiparticles to particles are approximately close to 1 and most of them is slightly less than 1, and the results of PYTHIA and PACIAE Model have good repeatability. The results obtained from our model are in agreement with the experimental data from STAR 21] and ALICE 22].

In order to study the energy dependence of the yield ratio of antiparticles to particles, the yield ratios of antiparticles to particles are calculated by the PACIAE and DCPC model for different center-of-mass (c.m) energies $p p$ collisions of $10 \mathrm{GeV}, 17.3 \mathrm{GeV}, 50 \mathrm{GeV}, 100 \mathrm{GeV}, 200 \mathrm{GeV}, 1 \mathrm{TeV}, 7 \mathrm{TeV}, 14 \mathrm{TeV}$, respectively, as shown in Figure 2 . The ratios for the $\bar{p} / p$ and $\bar{\Lambda} / \Lambda$ are obtained by PACIAE model, the ratios for the $\bar{d} / d, \overline{{ }^{3} H e} /{ }^{3} H e$, and $\frac{\overline{3}}{\Lambda} H / \frac{3}{\Lambda} H$ are calculated by PYTHIA + DCPC model for all energies.

We can see from the Figure 2 that the yield ratios ascend rapidly with the rise of c.m energy. When c.m energy reaches beyond $200 \mathrm{GeV}$, the yield ratios tend to saturate, and then the value of yield ratios of all particles gradually tend to 1 as the c.m energy is more than $1.0 \mathrm{TeV}$. It can also be noticed that the larger the mass of the particle is, the smaller the ratio is. In low energy, collisions frequency is relatively small, and the production cross section of final state particles coalesced by initial quark-antiquark pairs is also small, especially the cross section of antiparticles. When the beam energy of the collisions is increased, the system created becomes almost net-baryon free [22]. It means that probability of antiparticles production will reach that of particles. This energy dependence of the yield ratio of antiparticles to particles in high $p p$ collisions is similar to the relativistic heavy ion collisions 22, 23.

The transverse momentum distributions of (anti)particles produced by PACIAE model for the different c.m. energies in $p p$ collisions are investigated, containing Pions, Kaons, Protons and Lambdas, respectively. The results at 


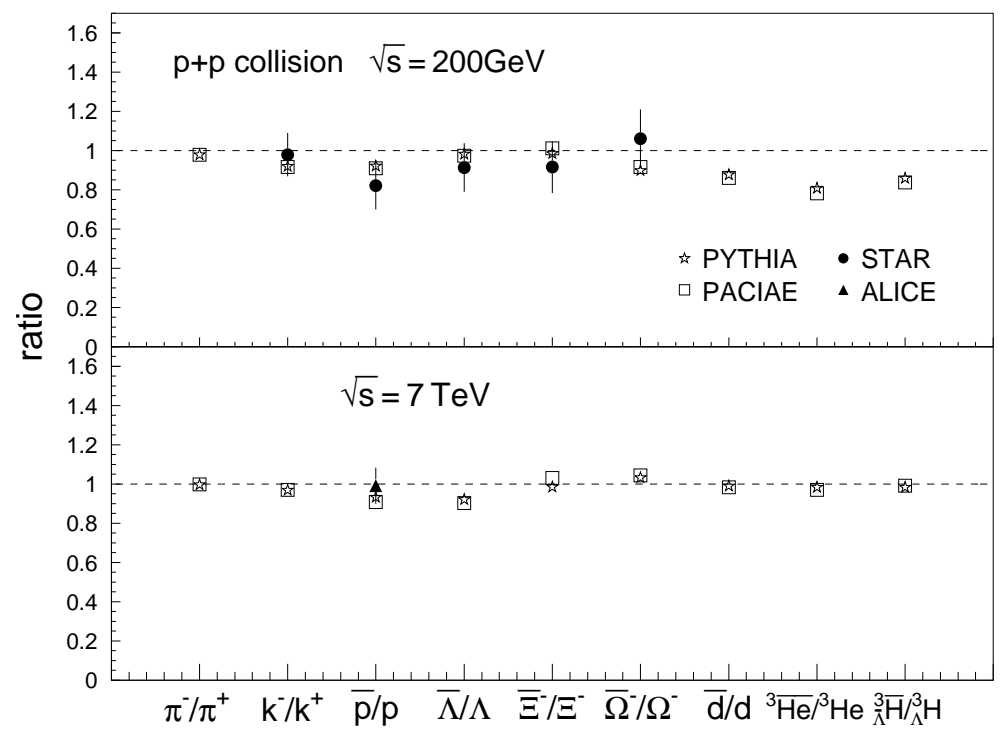

FIG. 1: The yield ratios of antiparticles $\left(\pi^{-}, K^{-}, \bar{p}, \bar{\Lambda}, \overline{\Xi^{-}}, \overline{\Omega^{-}}, \bar{d}, \overline{{ }^{3} H e}\right.$, and $\left.\overline{\frac{3}{\Lambda} H}\right)$ to corresponding particles $\left(\pi^{+}, K^{+}, p\right.$, $\Lambda, \Xi^{-}, \Omega^{-}, d,{ }^{3} \mathrm{He}$, and $\left.\frac{3}{\Lambda} H\right)$ in $p p$ collisions of $200 \mathrm{GeV}$ and $7 \mathrm{TeV}$, where mesons and Baryons produced by PYTHIA and PACIAE Models, the light (anti)nuclei and (anti)hypertritons produced by DCPC model. The open symbols represent our model results. The solid symbols are the data points from STAR ${ }^{21}$ and ALICE ${ }^{22}$.

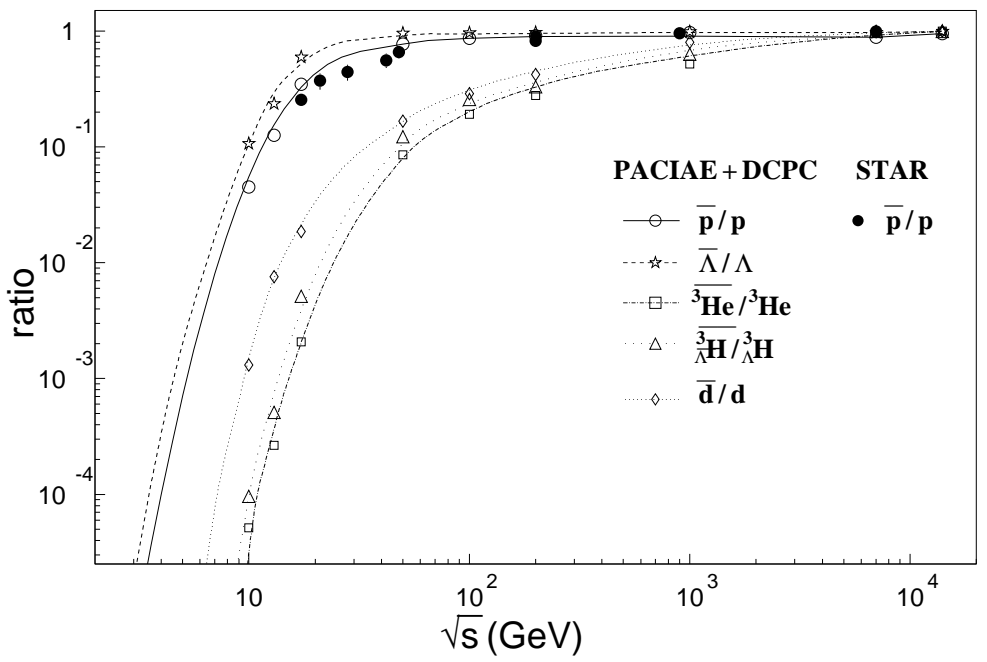

FIG. 2: The distribution of yield ratios of antiparticles $\left(\pi^{-}, K^{-}, \bar{p}, \bar{\Lambda}, \overline{\Xi^{-}}, \overline{\Omega^{-}}, \bar{d}, \overline{3} H e\right.$, and $\left.\overline{\frac{3}{\Lambda} H}\right)$ to corresponding particles $\left(\pi^{+}, K^{+}, p, \Lambda, \Xi^{-}, \Omega^{-}, d,{ }^{3} H e\right.$, and $\left.\frac{3}{\Lambda} H\right)$ in $p p$ collisions, as a function of c.m energy, which the data are simulated by PACIAE + DCPC model. The c.m energies are $10 \mathrm{GeV}, 13 \mathrm{GeV}, 17.3 \mathrm{GeV}, 50 \mathrm{GeV}, 100 \mathrm{GeV}, 200 \mathrm{GeV}, 1 \mathrm{TeV}, 7 \mathrm{TeV}$, and $14 \mathrm{TeV}$ respectively.

$\sqrt{s}=17.3 \mathrm{GeV}, 1000 \mathrm{GeV}$ and $14000 \mathrm{GeV}$ are shown in figure 3 . From the figure 3 , one can observe that the transverse momentum distributions increase sharply from $p_{T}=0 \mathrm{GeV} / \mathrm{C}$ until a maximum peak around $p_{T}=0.2 \sim 0.5 \mathrm{GeV} / \mathrm{C}$ is reached. For one particle, the peak value of transverse momentum distributions reduces with the c.m. energy increasing and distribution is broadened. The transverse momentum distributions of different particle, shown in Figures 3(a)-(d), have a similar behavior, which the peak moves rightward with the increasing of mass of particle. However, the transverse momentum distributions of antiparticles have a similar behavior with particles, whereas the peak of antiparticle distributions moves leftward and the becomes narrower compared to the particles distributions.

Figure 4 shows the energy dependence of average transverse momentum of different particles, including antiparticles correspondingly. It can be seen from the figure that the average transverse momentum increases with the 


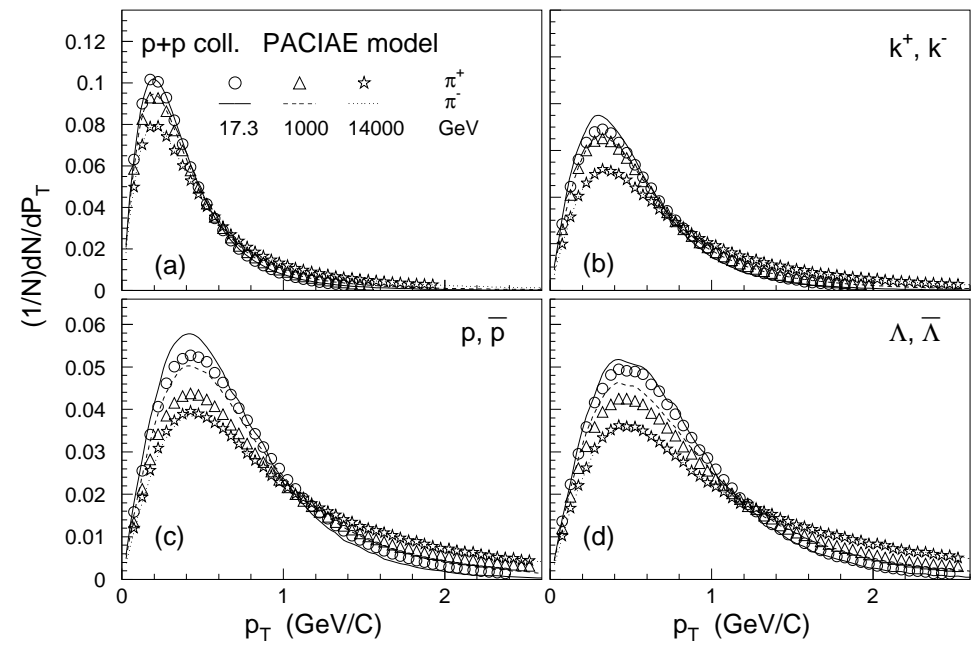

FIG. 3: The transverse momentum distributions of particles and anti-particles produced by PACIAE model at $\sqrt{s}=17.3 \mathrm{GeV}$, $1000 \mathrm{GeV}$ and $14000 \mathrm{GeV}$ in $p p$ collisions, respectively, as a function of c.m energy. (a) $\pi^{+}, \pi^{-}$, (b) $K^{+}, K^{-},(\mathrm{c}) p, \bar{p},(\mathrm{~d}) \Lambda, \bar{\Lambda}$.

rise of energy. It can be seen from figure 3 that the amount of particle in high $p_{T}$ region would increase with higher energy. So we suggest that the rise of energy leads to more violent collisions, larger transferring of momentum and energy, and then larger cross section of hard process and hard particle production, which obviously would raise the average transverse momentum. Besides, we notice that the average transverse momentum of mesons is smaller than that of baryons. It can be seen from figure 3 that the larger the mass of the particle is, the more the amount of particle in high $p_{T}$ region is, then the larger the average transverse momentum is. Since the mass of mesons is less than the mass of baryons, the corresponding average transverse momentum of mesons is smaller than that of baryon. Actually, the average transverse momentum of particles is mass dependent, i.e. the transverse momentum increases with the increasing of mass, this conclusion is just consistent with the experimental results [21].

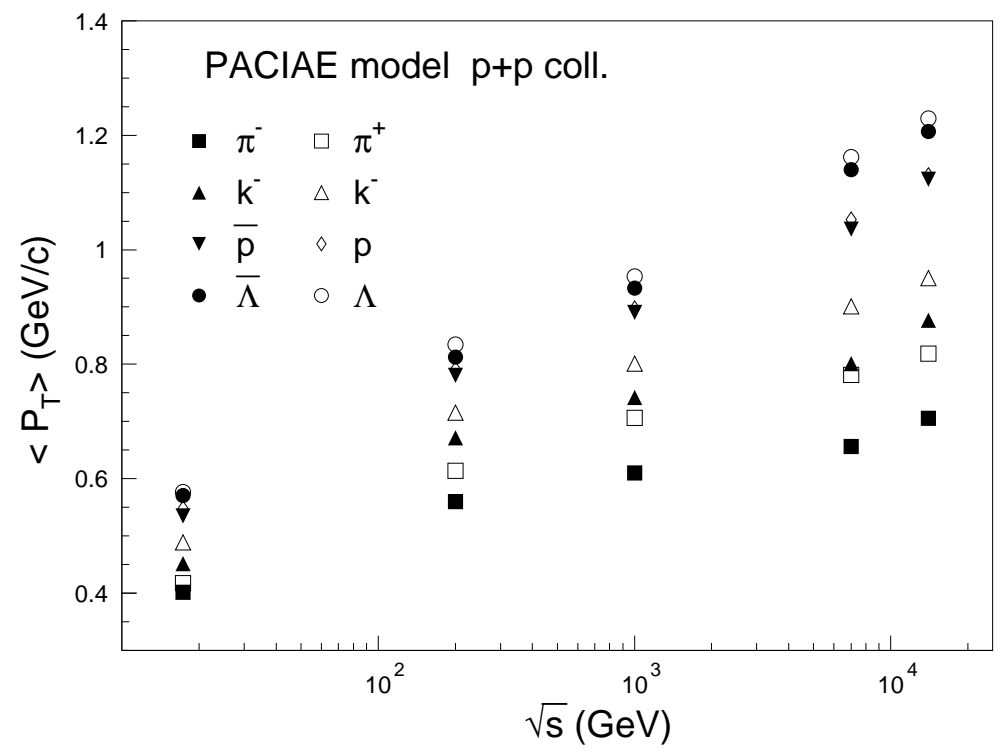

FIG. 4: The values of average transverse momentum for different particles $\left(\pi^{-}, K^{-}, \bar{p}, \bar{\Lambda}, \pi^{+}, K^{+}, p, \Lambda\right)$ in $p p$ collisions, as a function of c.m energy, which the data are simulated by PACIAE model. The c.m energies are $17.3 \mathrm{GeV}, 200 \mathrm{GeV}, 1 \mathrm{TeV}$, $7 \mathrm{TeV}$, and $14 \mathrm{TeV}$ respectively. 


\section{CONCLUSION}

We use PACIAE and PYTHIA model to generate final state hadrons of different c.m energies in pp collisions and combine the light (anti-)nuclei with the DCPC model. Firstly, the energy dependence of antiparticle $\left(\pi^{-}, K^{-}, \bar{p}, \bar{\Lambda}\right.$, $\overline{\Xi^{-}}, \overline{\Omega^{-}}, \bar{d}, \overline{{ }^{3} H e}$, and $\left.\overline{\frac{3}{\Lambda} H}\right)$ to particle $\left(\pi^{+}, K^{+}, p, \Lambda, \Xi^{-}, \Omega^{-}, d,{ }^{3} H e\right.$, and $\left.\frac{3}{\Lambda} H\right)$ ratios in high energy $p p$ collisions is studied. The results show that antiparticle to particle ratios increase with the increase of the c.m energy of $p p$ collisions from $\sqrt{s}=10 \mathrm{GeV}$, until they gradually approach to 1 after the c.m energy is more than $200 \mathrm{GeV}$. Then the distribution of transverse momentum of antiparticles and particles at different c.m energies are investigated. It is found that the transverse momentum distributions of different particles at different c.m energies have a similar behavior, for one particle its peak reduce and the distribution broaden with the increasing of the c.m energy of particle. However, its peak moves rightward with the increasing of mass of particle at same c.m energy. Contrary, the average transverse momentum increase when the c.m energy of $p p$ collisions increase. The model results are compatible with the STAR preliminary datum.

\section{ACKNOWLEDGMENT}

Finally, we acknowledge the financial support from NSFC $(11475149,11305144,11303023)$ in China.

[1] Yan Yuliang et al., Phys. Rev. C 85 (2012) 024907.

[2] P. A. M. Dirac, Proc. Cambridge Phil. Soc 26 (1930) 361.

[3] C. D. Anderson, Phys. Rev. 43 (1933) 4912494.

[4] O. Chamberlain et al., Lawrence Berkeley National Laboratory, 1955.

[5] B. Corket et al., Phys. Rev. 104 (1956) 1193.

[6] The ALPHA Collab., Nature 468 (2010) 673-676.

[7] ALICE Collab. (N. Sharma et al.), Phys. Rev. Lett. 106 (2011) 032301.

[8] ALICE Collab. (N. Sharma et al.), Nuclear and Particle Physics 38 (2011) 124189.

[9] R. Mattiello et al., Phys. Rev. C 55 (1997) 1443-1454.

[10] V. Topor Pop et al., Phys. Rev. C 81 (2010) 054911.

[11] Li Feng et al., Phys Rev C 85 (2012) 064912.

[12] J. Steinheimer et al., Phys. Lett. B 714 (2012) 85-91.

[13] Yan Yuliang et al., Phys. Rev. C 81 (2010) 044914; Sa Benhao et al., Phys. Commun. 183 (2012) 333.

[14] Chen Gang et al., Phys. Rev. C 86 (2012) 054910.

[15] Chen Gang et al., Phys. Rev. C 88 (2013) 034908.

[16] Chen Gang et al., arXiv:1401.6872v1.

[17] T. Sjostrand et al., High Energy Phys. 05 (2006) 026.

[18] B. L. Combridge et al., Phys. Lett. B 70 (1977) 234.

[19] Sa Benhao et al., Comput. Phys. Commun. 90 (1995) 121; Tai An et al., Comput. Phys. Commun. 116 (1999) 353.

[20] K. Stowe (Cambridge University Press, Cambridge, UK, 2007); R. Kubo et al., Statistical Mechanics (North-Holland, Amsterdam, 1965).

[21] STAR Collab. (B. I. Abelev et al.), Phys. Rev. C 75 (2007) 064901.

[22] ALICE Collab. (K. Aamodt et al.), Phys. Rev. Lett. 105 (2010) 072002.

[23] A. Andronic et al., Phys. Lett. B 697 (2011) 203-207. 